\title{
Quasicrystalline Ordering in Thin Liquid Crystal Films
}

\author{
Jayasri Dontabhaktuni ${ }^{1,+}$, Miha Ravnik ${ }^{2,3,+}$ and Slobodan Zumer ${ }^{2,3, *,+}$ (iD \\ School of Natural Sciences, Mahindra Ecole Centrale, Hyderabad 500043, India; jayasri.d@mechyd.ac.in \\ 2 Faculty of Mathematics and Physics, University of Ljubljana, 1000 Ljubljana, Slovenia; \\ miha.ravnik@fmf.uni-lj.si \\ 3 Josef Stefan Institute, 1000 Ljubljana, Slovenia \\ * Correspondence: slobodan.zumer@fmf.uni-lj.si; Tel.: +386-1-4766-657 \\ + These authors contributed equally to this work.
}

Received: 31 March 2018; Accepted: 25 June 2018; Published: 29 June 2018

\begin{abstract}
Quasicrystalline ordering was first observed in synthetic multi-component metallic alloys. These solid state materials exhibit quasicrystalline atomic ordering at nanometer length scales. Softmatter systems are another class of versatile materials that can exhibit quasicrystalline ordering across supra-nanometer $(>10 \mathrm{~nm})$ to supra-micrometer $(>10 \mu \mathrm{m})$ length scales as recently observed in materials like-supramolecular dendritic molecules, $\mathrm{ABC}$ star polymers, binary nanoparticle systems and block co-polymers in condensed matter systems. The underlying mechanism in most of these soft quasicrystals seems to be the presence of two or more length scales in the system. Another class of development in self-assembled quasicrystals in softmatter is being observed in low molecular weight chiral and achiral nematic liquid crystals. Liquid crystal forms an efficient matrix for selfand directed-assemblies of colloidal structures where surface and geometry-tuning the particles in nematic liquid crystals gives rise to complex inter-particle interactions while the long-range order results in self-assembled structures of higher order rotational symmetries. Furthermore, there has also been attempts to generate colloidal quasicrystalline defect structures by directing the assemblies using multiple and single beam lasing techniques. In the present article, we will review self- and assisted-assembly of quasicrystalline structures in nematic liquid crystals (both chiral and achiral) and discuss the underlying mechanisms.
\end{abstract}

Keywords: liquid crystals; nematic; cholesteric; quasi crystals; self-assembly; topological defects; colloids; optical manipulation

\section{Introduction}

Quasicrystalline ordering has been first observed in synthetic multicomponent metal alloys governed by an irrational ratio of two or more atomic length scales [1,2]. Natural solid metal alloys with quasicrystalline structures were found [3]. Nearly 20 years ago the theoretical predictions based on monoatomic liquids [4] and charged colloidal dispersions in deionized solvent [5] indicated the existence of stable quasicrystalline phases and their approximants. This was followed by Monte Carlo simulations [6] on spherical particles with hard core and soft repulsive shoulder which gave rise to stable single component quasicrystals. Few years later it was discovered that the self-assembly of dendrimers leads to stable soft matter with quasicrystalline ordering $[7,8]$. The cone-like molecules form spherical supra-molecules Figure 1a,b that further self-organize into different three-dimensional crystalline structures including quasicrystal (Figure 1d) which exhibits dodecagonal rotational symmetry normal to the rotation axis while exhibiting periodic order along the rotation axis. The quasicrystalline tilings were modelled using two basic prototiles-triangular and square shaped elements (Figure 1c) made of the spherical supramolecules at different layers 
along the rotation axis. This opened the sequence of observation of quasicrystals based on star terpolymers [9,10], binary nanoparticle systems [11], copolymers in melts [12], micellar colloids [13,14] and hard tetrahedra [15]. Formation of these structures in soft matter has been enabled by the advances in the chemical synthesis and nano material processing techniques. Typically, these patterns in softmatter systems cover a range of length scales from supra-nano to supra-micron sizes compared to the intermetallic systems which occur in nanometer range. Theoretically, the quasicrystalline ordering in softmatter is addressed using pair potentials with hard core and a soft repulsive shoulder interactions [16-19]. The growth of quasicrystalline long-range ordering from simple periodic approximants interacting via short-range potentials were also studied using molecular simulations [20-22].

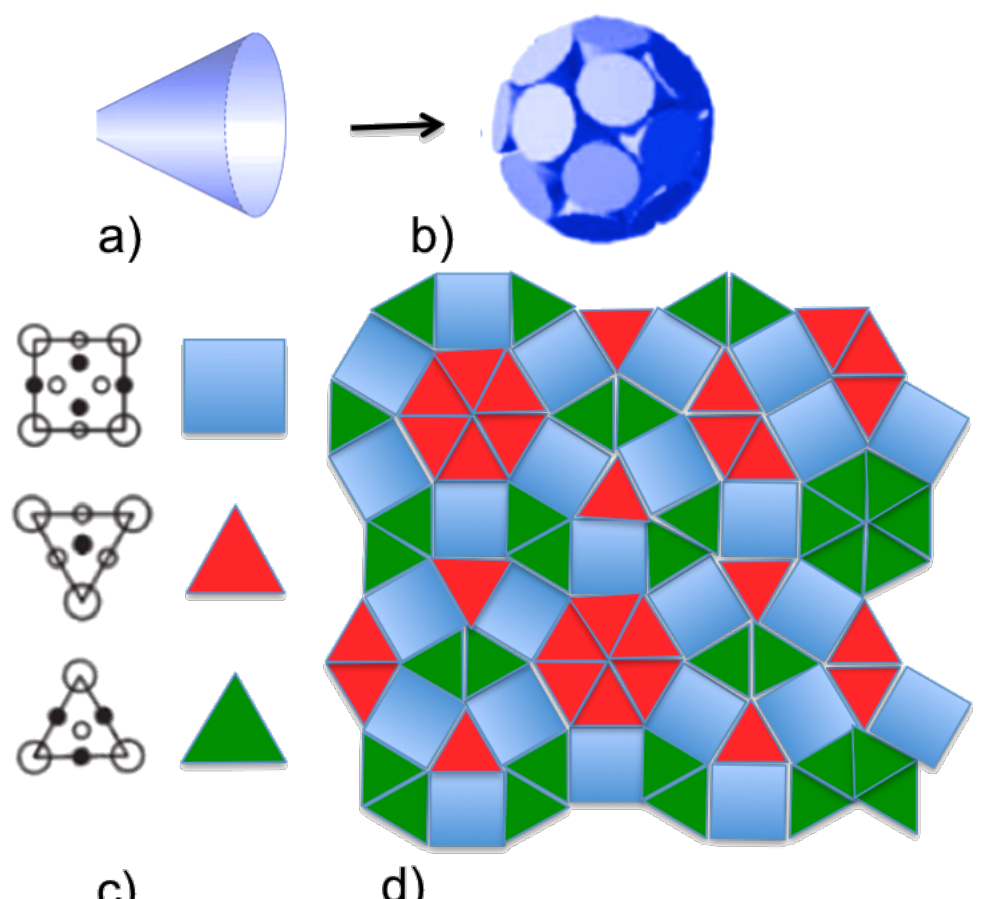

c)

d)

Figure 1. Schematic representation of the spherical supramolecules formed from the cone-shaped dendrimers and the quasicrystalline structures normal to the rotation axis. (a,b) Cone shaped dendrimers assemble into spherical supramolecules. (c) Basic structural elements decorated with spherical micelles at different elevations along the rotation axis and prototiles represented schematically as simple squares and triangles. (d) Quasicrystalline structures as seen in the plane normal to rotation axis. For more details see $[7,8]$.

Relevance of geometry of the constituent colloidal particles and the presence of multiple length scales in the softmatter systems are identified as crucial mechanisms for the formation of quasicrystalline structures. The effect of geometry was studied in detail by the self-assembly of faceted platelets in a nematic layer [23-25] and tuning them to form quasicrystalline structures [26,27]. It is observed that geometry of colloidal particles dictates the symmetry of interactions induced in the nematic medium and hence the long-range ordering of the particles [23]. The symmetry of such interactions can be further tuned to give rise to quasicrystalline structures. In parallel, micron scale quasicrystalline patterns are induced by complex optical fields by trapping of colloidal particles [28], patterned photopolymerization of monomers dispersed in liquid crystals [29], and chiral nematics by creating an array of defects [30]. The recent developments of generating complex light beams and optical manipulation techniques helped in successfully assembling these structures. Characteristic scales of soft quasicrystalline patterns are often in the range of the visible light wave lengths. Therefore, 
perspectives of optical applications stimulated advances in this field, including formation of materials with large near-complete photonic bandgaps [31] and miniature lasers [29].

In this short review, we focus on liquid crystal-based quasicrystalline structures where anisotropic media enable specific colloidal and defect interactions. We will present and discuss the quasicrystalline structures predicted [26] and experimentally realized [27,32] in thin layers of achiral and chiral nematic liquid crystals by self-organization of faceted colloidal particles or by inducing array of defects with complex optical fields. Finally, we briefly discuss the role of symmetry of the colloidal particles in nematic liquid crystal to form quasicrystalline tilings in 2D.

\section{Quasicrystalline Ordering of Shaped Particles in Thin Nematic Layers}

Nematic liquid crystals have proven to be an efficient matrix for self and directed-assembly of colloidal structures. Nematic liquid crystals are anisotropic fluids characterized by orientational order. Dispersing colloidal particles into the nematic frustrates the nematic ordering surrounding the particles leading to topological defects. For example, spherical particles with homeotropic anchoring lead to the formation of dipolar structure with a hyperbolic -1 point defect or Saturn ring (quadrupolar) structure with $\mathrm{a}-1 / 2$ disclination line (Figure $2 \mathrm{a}, \mathrm{b}$ ). In case of degenerate planar anchoring the spherical particles give rise to structures with two surface hyperbolic boojum defects (Figure 2c). The parameters anchoring strength $(\mathrm{W})$, particle radius $(\mathrm{R})$, nematic elastic constant $(\mathrm{K})$, enter in the ratio $\mathrm{RW} / \mathrm{K}$ that is approximately equal to one when the structures have the same free energy [33]. When more particles are introduced in the medium, minimizing the elastic distortion energy of the nematic leads to effective interactions, that are strongly anisotropic as characterised by specific attractive and repulsive directions. For example, particles accompanied by dipolar or quadrupolar director fields dispersed in a thin nematic layer form homogenous $2 \mathrm{D}$ oblique lattices as seen in the Figure 2d-f [34]. In the case of homeotropic anchoring, disclinations can also form braids that can entangle two or more particles [35] and also result in knots and links [30,36] particularly in chiral nematics.

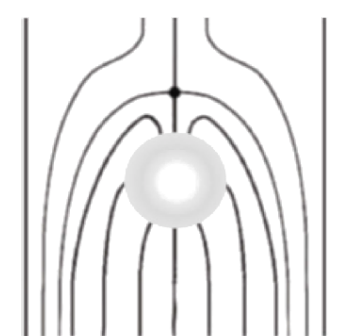

a)

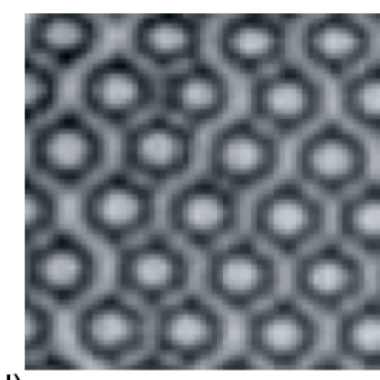

d)

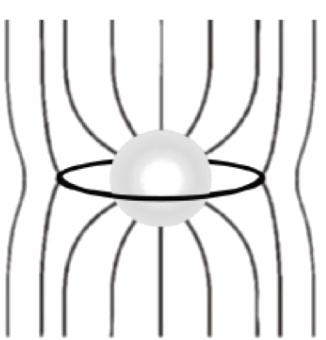

b)

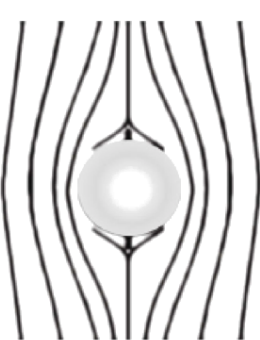

c)

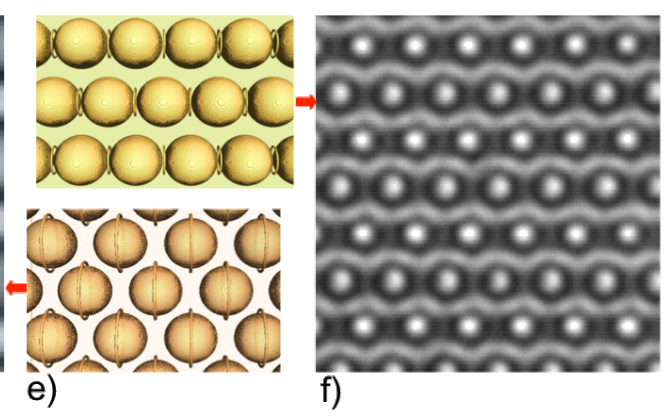

Figure 2. Defect configurations surrounding a spherical colloidal particle and stable 2D crystalline lattices. (a) Schematic of a point defect of strength -1 (dipole) and (b) disclination loop with winding number $-1 / 2$ (quadrupole) encircling colloidal particle with homeotropic anchoring. (c) Schematic of boojums on the colloidal particle with planar degenerate anchoring. (d) Polarization micrographs of stable crystalline lattices formed by quadrupolar units. (e) Simulation results of the corresponding quadrupolar and dipolar crystalline structures. (f) Polarization micrographs of stable crystalline structures of dipolar units. 
With the availability of advanced synthesis methods through chemical routes, the colloidal particles can have patterned anchoring [37] or have various shapes and geometries like polygons [23,24,27], handled-bodies [38], fractal structures [39], lock and key partices [40], etc. which in turn expand the possibilities of the self-assembled structures that form in liquid crystals. When polygonal platelets like square, triangular and pentagonal geometries are introduced in nematic, they give rise to director configurations depending on the geometry of the particles. It is observed that even-sided platelets give rise to quadrupolar symmetries while odd-sided platelets give rise to dipolar symmetries independent of the size of the particles $[23,24]$ as seen from the schematics Figure 3a,b. This odd-even behaviour is the result of the mirror symmetry breaking (or not breaking) of the director field surrounding such polygonal platelets.
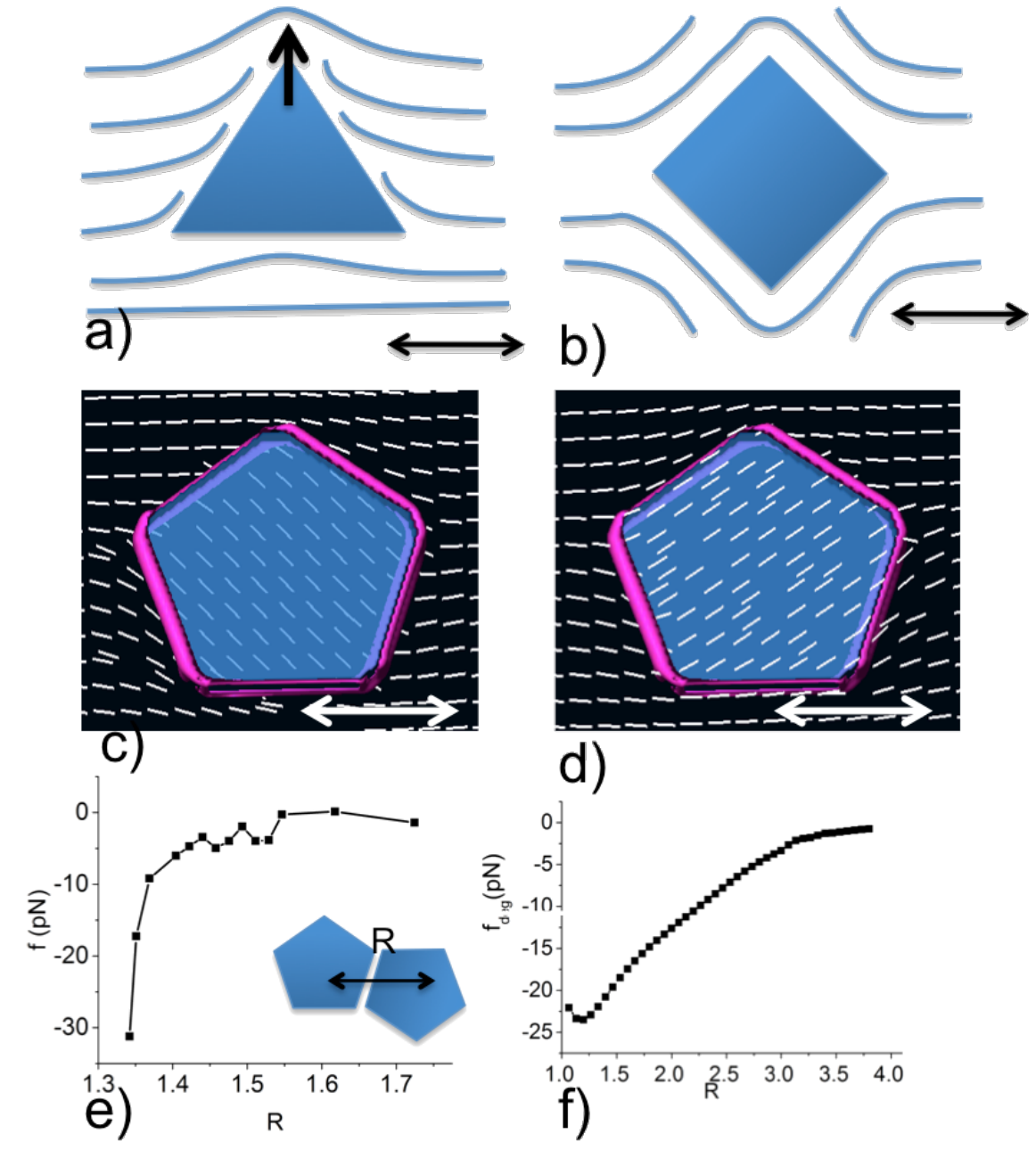

Figure 3. Faceted colloidal platelets in nematic layer. $(\mathbf{a}, \mathbf{b})$ Schematics of director field surrounding the triangular and square faceted platelets in nematic layer. (c,d) The pentagonal platelet (in blue) and the disclination lines surrounding the particles (in red). The disclination lines are visualized as isosurfaces of nematic order parameter corresponding to the $5 \%$ drop below its bulk value at $S=0.52$. The vectors represent the director field surrounding the platelets. The top face of the platelet has surface anchoring at an angle $36^{\circ}$, while the anchoring direction of the bottom face is $126^{\circ}$. (e) The inter-particle forces as a function of distance between the particles (in units of edge length, a of the particle) in the case of uniform anchoring at two different directions on the top and bottom faces. (f) Inter-particle forces in the case of degenerate anchoring on all the faces. 
It has been observed that pentagonal platelets with degenerate planar anchoring act as elastic dipoles and assemble into crystalline symmetries with rectangular unit cells [25]. In [26] the authors have reported that while such particles give rise to long range interactions with uniform surface anchoring, symmetry-breaking about one of the mid-plane of the particles gives rise to additional short range interactions which can be tuned to form self-assembled structures which are otherwise not possible with such platelets. The pentagonal platelets with uniform planar surface anchoring at an angle $\theta=36^{\circ}$ on the top face and $\theta=126^{\circ}$ on the bottom face of the platelet are considered as shown in the Figure $3 \mathrm{c}, \mathrm{d}$. The short range inter-particle attractive forces between the platelets with such a broken symmetry as a function of the lateral distance is shown in the Figure 3e. It is observed that there is an increase in the force as the distance between the particles is about $a=1.25 R$. The elastic distortion due to thin layer of the nematic in between the side faces of the platelets becomes comparable to the surface anchoring energy at this distance giving rise to an increase in the attractive forces. In comparison, the inter-particle attractive forces vary gradually as the separation increases in the case of degenerate anchoring as shown in the Figure $3 \mathrm{f}$.

Methodologically, a strong approach to describe and explore colloids in nematic complex fluids is to use minimization of the Q-tensor based Landau-de Gennes free energy, as described in detail in $[25,26]$. The approach is phenomenological and is based on the construction of invariants of the nematic order parameter tensor that cover basic ordering phenomena of the nematic: effective elasticity of the orientational order, the degree of orientational order and coupling with surrounding surfaces (surface anchoring). In one elastic constant approximation, the total nematic free energy can be written as:

$$
F=\quad \int_{L C}\left(L\left(\partial Q_{i j} / \partial x_{k}\right)^{2} / 2+\frac{1}{2} A Q_{i j} Q_{j i}+\frac{1}{3} B Q_{i j} Q_{j k} Q_{k i}+\frac{1}{4} C\left(Q_{i j} Q_{i j}\right)^{2}\right) d V+\int_{\text {surf }} f_{S} d S,
$$

where $L$ is the single elastic constant. Nematic material parameters are given by $A, B, C$ and $f_{S}$ gives surface free energy density that accounts for perpendicular, planar or other surface anchoring of nematic. Double appearances of indices in Q-tensor products and its derivatives stands for summation over those indices. $\int_{L C}$ performs the integral over the volume occupied by the liquid crystal and $\int_{\text {surf }}$ indicates the integral over the surfaces of the particle and the cell boundaries. The free energy is typically minimised with different numerical approaches, including finite difference relaxation, Lattice Boltzmann, finite elements and finite volumes method.

Penrose tiling is assembled in the bottom-up approach by spatially arranging basic repetitive units called prototiles in the form of rhombus, boat and star-shaped colloidal clusters. Defect lines are formed along the edges of the particles as well as in the gaps between the particles in the nematic. The stable configurations of the prototiles along with the disclination lines are shown in the Figure 4a. These prototiles are stable about in-plane rotations and have multiple minima in the free energy as shown in the article [25]. The stretching modes of these prototiles are also studied systematically as a function of orientation with the rubbing direction in Figure 4 , as selectively shown elementary channels for the diss-assembly of the prototiles. It is observed that the prototiles are stable against both symmetric and asymmetric stretching at all angles as shown in Figure $4 \mathrm{~b}-\mathrm{e}$. The stable Penrose tiling with 176 particles arranged in two dimensions with 5-fold symmetry is shown in Figure 5a.

Symmetry breaking can also be achieved by changing the geometry of the particles. With the advanced lithographic techniques it is possible to fabricate particles of asymmetric shapes. In [27], the truncated polygonal pyramid particles were fabricated and the self-assembled structures were studied. In the case of pentagonal pyramidal structure, one of the large faces is smaller than the other. The surface anchoring is normal to the the faces and the rubbing direction is perpendicular to the cell. As shown in the Figure 5b, these particles stabilize normal to the rubbing direction in the mid-plane of the cell with disclination lines of strength $s=-1 / 2$ forming along the larger face of the platelet. It is observed that these particles give rise to long-range dipolar interactions and short-range 
geometry dependent interactions. These platelets have side-to-side attractive forces, exhibiting dipolar nature. In a planar cell, the particles form assemblies along base-to-base separations with their faces normal to the rubbing direction. However, the base-to-base attractions are much weaker compared to the side-to-side attractions. As these particles are released using a laser tweezer, due to the short range interactions guided by the local geometry, these particles form quasicrystalline structures as shown in the Figure $5 \mathrm{c}$ locally reducing the elastic distortions. Since the binding energies due to these many-body interactions are much higher than the thermal fluctuations, these colloidal structures remain stable once formed. These two cases elucidate the role of symmetry of the particle interactions locally with the surrounding nematic in obtaining self-assembled quasicrystalline structures while globally retaining the long-range effective multipolar (electrostatic-like) interactions.
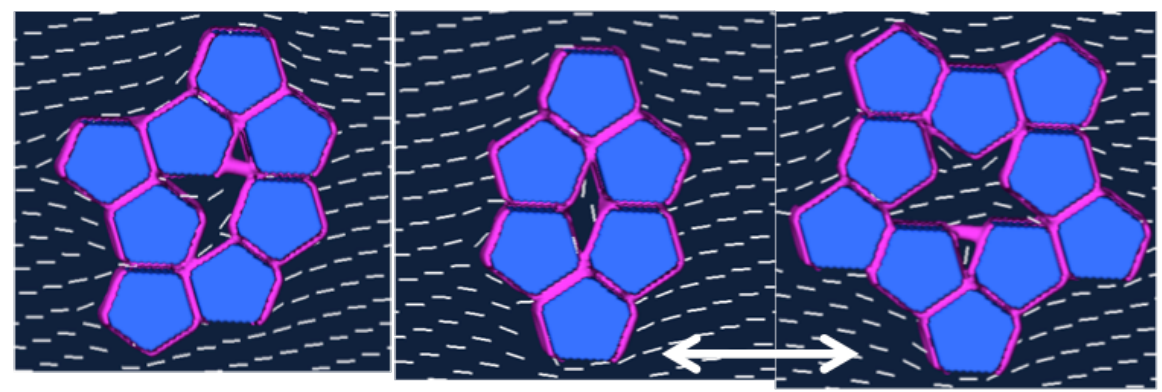

a)
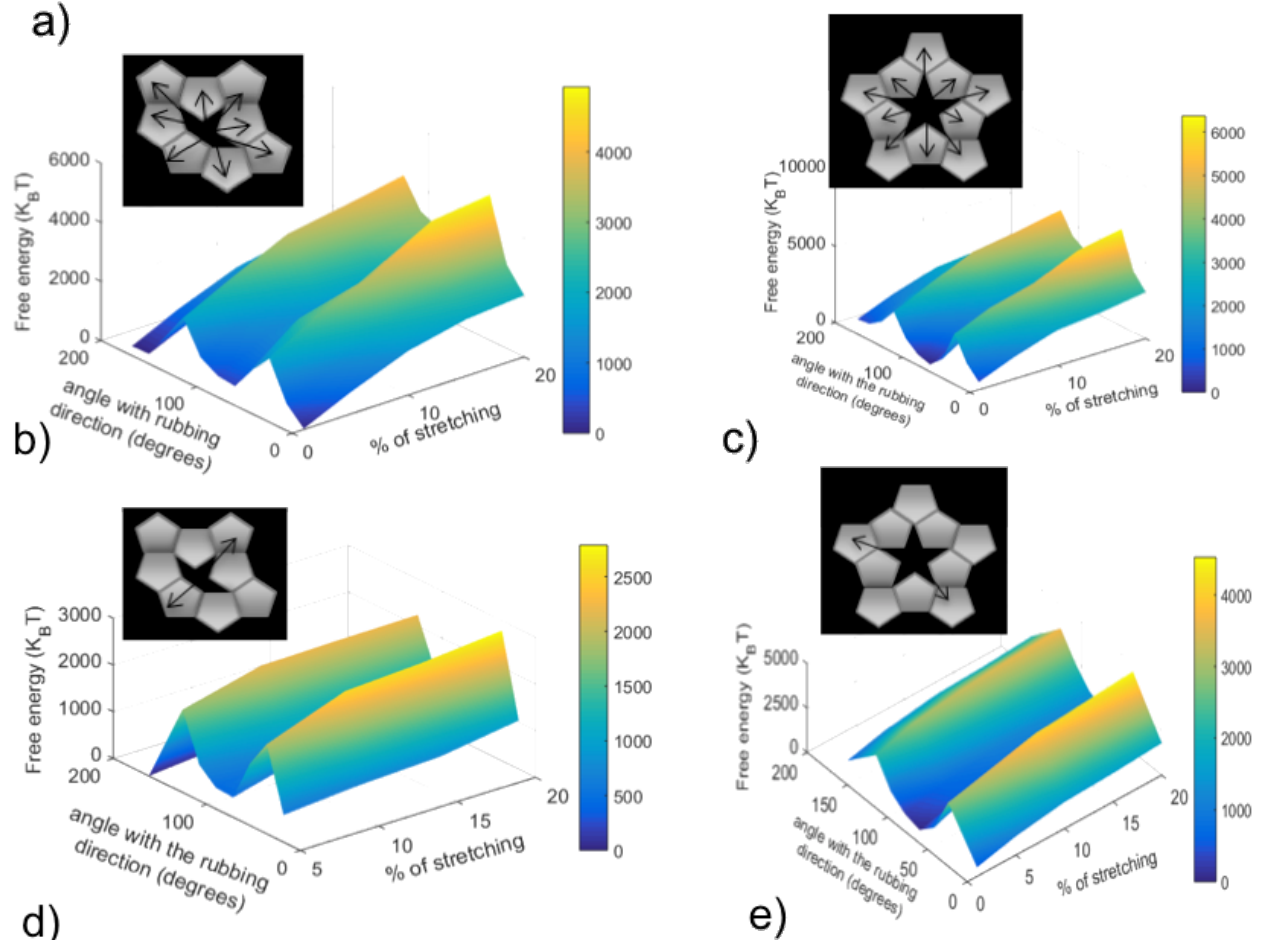

c)

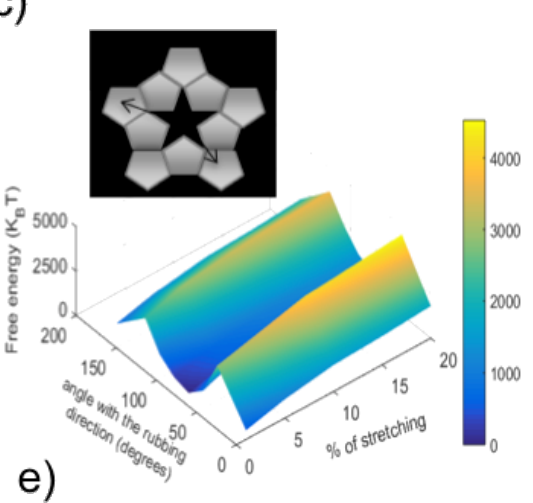

Figure 4. Stability of colloidal prototiles for Penrose P1 tiling. (a) Stable configurations of the basic prototiles-boat, rhombus and star. $(\mathbf{b}, \mathbf{c})$ Free energy profiles of the boat and star prototiles by symmetrically stretching them as shown in the insets. (d,e) Free energy profiles of the boat and star prototiles by the corresponding asymmetric stretching as shown in the insets. For further details, see [26]. 


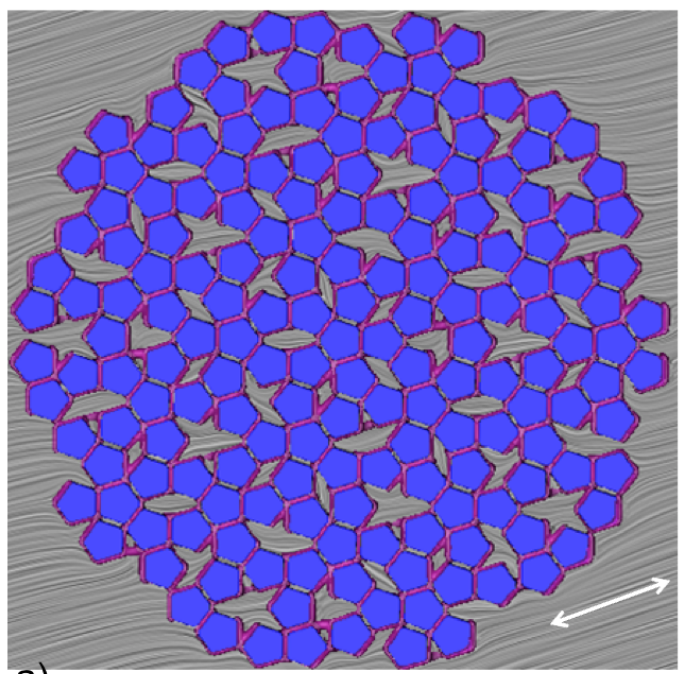

a)
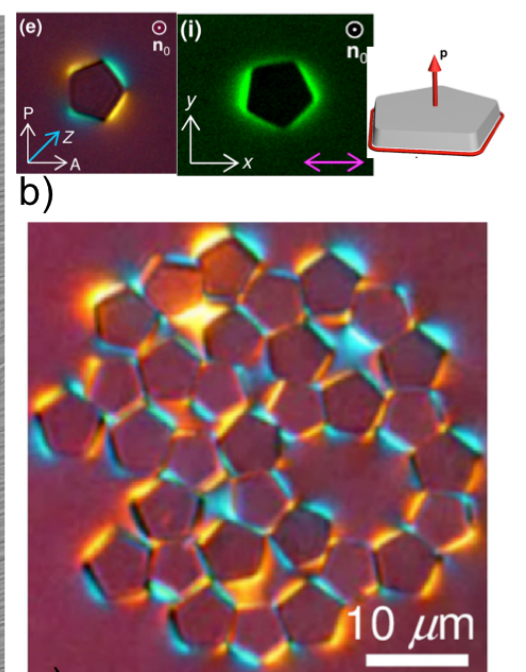

C)

Figure 5. (a) The Penrose tiling assembled from 176 pentagonal platelets. (b) POM images of polygonal platelets truncated on one face. Truncated polygonal platelet with the disclination line surrounding the edge in the inset. (c) Penrose tiling formed from the truncated polygons. For further details, see [26,27].

\section{Optically Induced Quasicrystalline Ordering of Particles in Thin Chiral Nematic Layers}

Controllability of stable defects in liquid crystals is conventionally performed either by local heating, applied fields or flow-induced tuning. In a recent line of work [32,41,42], authors have reported certain laser tweezing and trapping techniques to generate vortex-like defects called bubbles or torons in chiral nematic liquid crystals (-simply cholesterics, as often used in literature). In this section we will review the laser assisted 2D assembling of torons that may lead to lattices with quasicrystalline symmetries. Cholesteric liquid crystal is confined to a homeotropic cell with thickness smaller than the pitch $\mathrm{p}$. This leads to frustration of the chiral orientational ordering, which results in homogenous unwound (uniform) director field normal to the confining surfaces of the cell (Figure 6a). When a high intense Laguerre-Gaussian (L-G) beam with azimuthal number $l=1$ and vortex-like polarization [43] is incident along the initially homogenous director, it is observed that above a threshold intensity the homogenous structure gets perturbed. The director reorients locally along the azimuthal direction normal to the initial homogenous director field (Figure $6 \mathrm{~b}$ ). Since a chiral nematic is used, tilting of the director is accompanied by twisting thus forming a double-twist structure looped on to itself around the incoming beam. Topological constrains imposed by homeotropic surface anchoring further contribute to the double twist structure and to two hyperbolic hedgehog defects [41] that may open into two $-1 / 2$ disclination loops as seen in Figure 6c ([43]). These complex topologically protected structures are torons [41]. Although these structures are metastable they cannot decay due to high elastic energy barrier presented by the topological and the geometrical constraints.

Modeling of the toron structures with point defects can be reasonably well addressed by minimizing Frank elastic energy based on simple director field description [41]. However, the light-induced structure formation and the appearance of singular disclination loops requires Q-tensor modeling based on the minimization of the Landau-de Gennes free energy. The simplest form used for nematic structures given in Equation (1) can be extended to describe toron formation in cholesterics by adding chiral and dielectric contributions as given below [44,45]:

$$
\begin{aligned}
F_{\text {chiral }} & =2 q_{0} L \int_{L C} \epsilon_{i j k} Q_{i j} \frac{\partial Q_{i j}}{\partial x_{k}} d V, \\
F_{\text {dielectric }} & =-\frac{1}{2} \int_{L C} \varepsilon_{0}\left(\bar{\varepsilon} \delta_{i j}+\frac{2}{3} \Delta \varepsilon^{m} Q_{i j}\right) E_{i} E_{j} d V,
\end{aligned}
$$


The chiral parameter $q_{0}$ is related to the pitch by $p=2 \pi / q_{0} . \varepsilon_{i k l}$ represents the Levi-Civita tensor in three dimensions. $\varepsilon_{0}$ represents the value of average dielectric constant and $\Delta \varepsilon^{m}$ represents the molecular dielectric anisotropy of the liquid crystal. $E_{i}$ and $E_{j}$ represent the electric field components of the vector E. Dielectric term can be used for both low frequency and optical E-fields if appropriate dielectric constants are used.
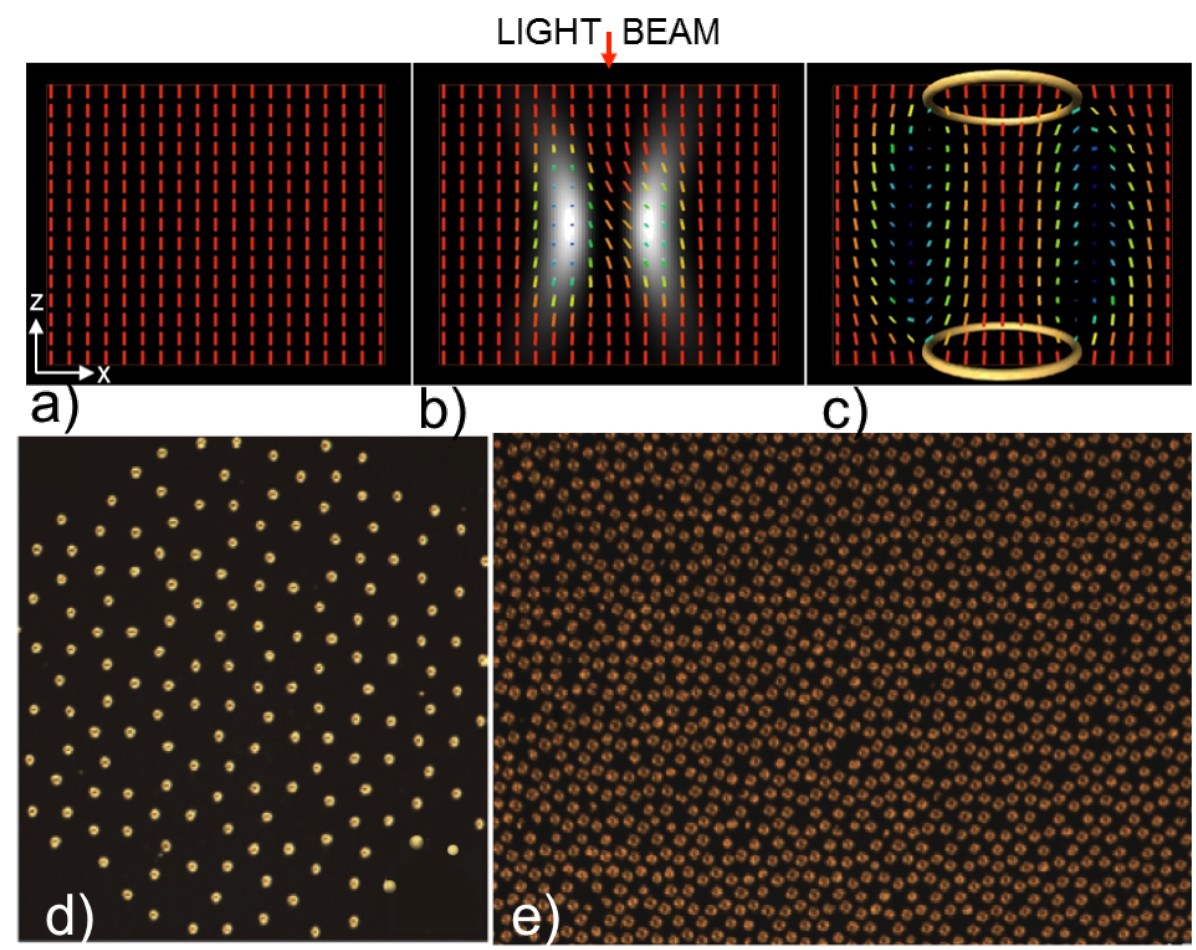

Figure 6. Simulated single toron profile and the experimental quasicrystalline structures formed by torons. (a) Simulated imprinting of a toron in the unwound cholesteric layer by the incoming $l=1 \mathrm{~L}-\mathrm{G}$ light beam along the homogenous director. Director fields in the plane of incoming beam illustrated by the colored bars where change of color from red to blue indicate out-of-plane reorientation of the director, length of the vector represents the amount of reorientation. (b) The director field along with regions of high intensity beam visualised in white. (c) Final toron structure with two $-1 / 2$ disclination loops represented by yellow iso-surfaces of the depressed nematic order parameter. For further details see [43]. (d,e) Polarizing microscope images of the quasicrystalline structures of torons. Obtained with permission from the author [41].

Controlled generation of periodic arrangement of these torons is performed using computer-programmed laser tweezing system [32,41]. The use of optical fields can also be complemented by application of low frequency electrical fields [42].The interaction between the torons is found to be repulsive in nature as the separation between them is less than the cholesteric pitch, $p$, hence the toron pair stabilizes at separations comparable to the $p$ giving rise to regular arrangement of the torons. Thus the periodic arrangement of the torons in cholesterics result in the formation of stable diffraction gratings of desired geometry. This precise control on generation of torons was exploited to generate two dimensional quasicrystalline structures as shown in the Figure $6 \mathrm{c}, \mathrm{d}$. The underlying mechanism in generating these structures resemble that of the hard core-soft corona potentials observed theoretically $[16,17,19]$. The stable periodic and aperiodic arrays of torons hence generated can be used as a stable diffraction gratings or as templates to assemble the colloidal particles into desired geometries with potential applications in photonics. 


\section{Discussion}

Development of novel soft matter materials, including with quasicrystalline symmetries can be strongly supported by theoretical and computer modelling approaches, to either guide or explain experiments. Soft matter systems generally span a broad phase and parameter space, and hence modelling and theory can prove invaluable in understanding these systems. An impressive example of such an approach is by Sharon Glotzer group, where they performed systematic studies of how shape of hard-core polyhedral particles affects the self-assembly and self organise into many-particle structures [46]. The study has shown organisation into disordered structures, liquid crystals, crystals, plastic crystals. The free energies described above for nematic and chiral nematic fluids serve as the main methodological approaches in complex nematic systems - at the usually experimentally explored mesoscales-for predicting or explaining the formation of particle or field structures, especially when used in combination with efficient numerical approaches.

The development of birefringent structures and profiles from nematic complex fluids, including structures and field profiles with quasicrystalline symmetries, indicates interesting potential for use in various complex optical and photonic elements, such as lasers, resonators, and waveguides. For example, blue phases and cholesteric LCs have demonstrated the capability to work as high-efficiency, tunable, paintable and white lasers [47-50]. Alternatively, nematic and chiral nematic structures can also perform as tunable photonic crystals [51,52]. Nematic droplets are shown to act as highly tunable optical resonators [53], whereas cholesteric droplets are shown to function as 3D omnidirectional micro-lasers [54]. Most recently, low-surface-tension tubular structures and colloidal tilings are used for the flow-of-light guiding and lasing $[55,56]$. These devices rely on the chemical incompatibility of the LC and the carrier fluid, which results in phase segregation and the formation of LC droplets and fibres with perfectly defined internal structure and optically perfect interfaces conditioned by the surface tension. WGM lasing can be tuned over large interval of $20 \mathrm{~nm}$ at $1.5 \mathrm{~V} / \mathrm{m}$ which demonstrates the potential of LCs to form an all-optic photonic platform. Fundamentally, the interaction between light and liquid crystals is mutual [57]. On one hand, the inherent (spatially and time dependent) birefringence of the nematic affects the flow-of-light whereas on the other hand, the dielectric coupling between the light field and the (induced) dipoles of the nematic molecules can change the birefringence profile of the nematic. Particularly, this coupling can become very relevant at strongly varying nematic profiles or at higher light intensities. The coupling between these two effects give rise to interesting phenomena such as nematicons [58], left-handed optical radiation torque [59], backwards torque [60], manipulation of orbital angular momentum of light [61] and coupling between topological defects in light fields and material fields [62]. Recently, chiral liquid crystal structures with a self-organized helical structure were shown to enable metasurface-like, non-specular reflection in the visible region [63], light induced liquid crystallinity was shown as a result of photo-induced conformational changes in molecular structure capable of yielding order-increasing phase transitions [64] and topological nanocolloids with facile electric switching of plasmonic properties were demonstrated [65]. To generalise, the notable advantages of soft matter approaches to photonics are: (i) inherent tunability of photonic elements with a variety of mechanisms, including electric, magnetic and light fields, (ii) self-assembly of the soft-matter structures making them available at larger scale, and (iii) generally smooth boundaries between soft (typically liquid) optical components as this is simply assured by the surface tension.

\section{Conclusions}

Assembly and generation of quasicrystalline structures in soft matter and specifically low molecular weight liquid crystalline systems is reviewed in short. We start our report with a brief listing of high molecular weight soft matter systems where quasicrystalline structures are produced by chemical synthesis in supra-molecular dendritic molecules, star terpolymers and block co-polymers. In contrast, a low molecular nematic liquid crystal is found to form an efficient matrix for the self-organized quasicrystals as colloidal interactions can be tailored either by geometrical manipulation 
or by changing the surface anchoring. Symmetry breaking of the nematic field imposed by polygonal platelets gives rise to two-dimensional quasicrystalline tilings. Extending this idea of symmetry breaking by geometry and by tailoring the surface anchoring can also give rise to quasicrystals in three dimensions [66]. Another novel route towards achieving the quasicrystalline structures is by optically generating vortices in the liquid crystal matrix which then act as building blocks to form two and three dimensional quasicrystalline structures. Generation of such softmatter-based quasicrystalline structures is motivated by possible applications in tunable optical elements like diffraction elements and tunable photonic crystals.

Author Contributions: The authors J.D., M.R. and S.Z. have contributed equally to this review.

Funding: Authors acknowledge funding from Slovenian Research Agency through Grants P1-0099, J1-7300, L1-8135, USAF AFRL EOARD research project Nematic Colloidal Tilings as Tunable Soft Metamaterials (Grant No. FA9550-15-1-0418) and Indian Research Funding Agency SERB-DST EMR Grant No. EMR/2017/004045.

Conflicts of Interest: The authors declare no conflict of interest.

\section{References}

1. Shechtman, D.; Blech, I.; Gratias, D.; Cahn, J. Metallic Phase with Long-Range Orientational Order and No Translational Symmetry. Phys. Rev. Lett. 1951, 53, 1951. [CrossRef]

2. Levine, D.; Steinhardt, P.J. Quasicrystals: A New Class of Ordered Structures. Phys. Rev. Lett. 1984, 53, 2477. [CrossRef]

3. Bindi, L.; Steinhardt, P.J.; Yao, N.; Lu, P.J. Natural Quasicrystals. Science 2009, 324, 1306-1309. [CrossRef] [PubMed]

4. Dzugutov, M. Formation of a dodecagonal quasicrystalline phase in a simple monatomic liquid. Phys. Rev. Lett. 1993, 70, 2924-2927. [CrossRef] [PubMed]

5. Denton, A.; Loewen, H. Stability of colloidal quasicrystals. Phys. Rev. Lett. 1998, 81, 469. [CrossRef]

6. Jagla, E.A. Minimum energy configurations of repelling particles in two dimensions. J. Chem. Phys. 1999, 110, 451-456. [CrossRef]

7. Zeng, X.; Ungar, G.; Liu, Y.; Percec, V.; Dulcey, A.E.; Hobbs, J.K. Supramolecular dendritic liquid crystals. Nature 2004, 428, 157-160. [CrossRef] [PubMed]

8. Ungar, G.; Percec, V.; Zeng, X.; Leowanawat, P. Liquid Quasicrystals. Isr. J. Chem. 2011, 51, $1206-1215$. [CrossRef]

9. Takano, A.; Kawashima, W.; Noro, A.; Isono, Y.; Tanaka, N.; Dotera, T.; Matsushita, Y. A mesoscopic Archimedean tiling having a new complexity in an ABC star polymer. J. Polym. Sci. Part B Polym. Phys. 2005, 43, 2427-2432. [CrossRef]

10. Hayashida, K.; Dotera, T.; Takano, A.; Matsushita, Y. Polymeric Quasicrystal: Mesoscopic Quasicrystalline Tiling in ABC Star Polymers. Phys. Rev. Lett. 2007, 98, 195502. [CrossRef] [PubMed]

11. Talapin, D.V.; Shevchenko, E.V.; Bodnarchuk, M.I.; Ye, X.; Chen, J.; Murray, C.B. Quasicrystalline order in self-assembled binary nanoparticle superlattices. Nature 2009, 461, 964-967. [CrossRef] [PubMed]

12. Lee, S.; Bluemle, M.J.; Bates, F.S. Discovery of a Frank-Kasper sigma phase in sphere-forming block copolymer melts. Science 2010, 330, 349-353. [CrossRef] [PubMed]

13. Fischer, S.; Exnera, A.; Zielskea, K.; Perlichb, J.; Deloudic, S.; Steurerc, W.; Lindnerd, P.; Forstere, S. Colloidal quasicrystals with 12-fold and 18-fold diffraction symmetry. Proc. Natl. Acad. Sci. USA 2011, 108, 1810-1814. [CrossRef] [PubMed]

14. Ungar, G.; Liu, Y.; Zeng, X.; Percec, V.; Cho, W.-D. Low-symmetry sphere packings of simple surfactant micelles induced by ionic sphericity. Science 2003, 299, 1208-1211. [CrossRef] [PubMed]

15. Haji-Akbari, A.; Engel, M.; Keys, A.S.; Zheng, X.; Petschek, R.G.; Palffy-Muhorray, P.; Glotzer, S.C. Disordered, quasicrystalline and crystalline phases of densely packed tetrahedra. Nature 2009, 462, $773-777$. [CrossRef] [PubMed]

16. Dotera, T.; Oshiro, T.; Ziherl, P. Mosaic two-lengthscale quasicrystals. Nature 2014, 506, 208. [CrossRef] [PubMed]

17. Dotera, T.; Bekku, S.; Ziherl, P. Bronze-mean hexagonal quasicrystal. Nat. Mater. 2017, 16, 987. [CrossRef] [PubMed] 
18. Ziherl, P.; Dotera, T. Soft Quasicrystals. J. Phys. Condens. Matter 2017, 24, 284101.

19. Ziherl, P.; Kamien, R.D. Maximizing Entropy by Minimizing Area Towards a New Principle of Self-Organization. J. Phys. Chem. B 2001, 105, 10147. [CrossRef]

20. Keys, A.S.; Glotzer, S.C. How do quasicrystals grow? Phys. Rev. Lett. 2007, 99, 235503. [CrossRef] [PubMed]

21. Iacovellaa, C.R.; Keys, A.S.; Glotzer, S.C. Self-assembly of soft-matter quasicrystals and their approximants. Proc. Natl. Acad. Sci. USA 2011, 108, 20935-20940. [CrossRef] [PubMed]

22. Engel, M.; Damasceno, P.F.; Phillips, C.L.; Glotzer, S.C. Computational self-assembly of a one-component icosahedral quasicrystal. Nat. Mater. 2015, 14, 109-116. [CrossRef] [PubMed]

23. Lapointe, C.P.; Mason, T.G.; Smalyukh, I.I. Shape-Controlled Colloidal Interactions in Nematic Liquid Crystals. Science 2009, 326, 1083. [CrossRef] [PubMed]

24. Lapointe, C.P.; Hopkins, S.; Mason, G.T.; Smalyukh, I.I. Electrically Driven Multiaxis Rotational Dynamics of Colloidal Platelets in Nematic Liquid Crystals. Phys. Rev. Lett. 2010, 105, 178301. [CrossRef] [PubMed]

25. Dontabhaktuni, J.; Ravnik, M.; Žumer, S. Shape-tuning the colloidal assemblies in nematic liquid crystals. Softmatter 2012, 8, 1657-1663. [CrossRef]

26. Dontabhaktuni, J.; Ravnik, M.; Žumer, S. Quasicrystalline tilings with nematic colloidal platelets. Proc. Natl. Acad. Sci. USA 2014, 111, 2464-2469. [CrossRef] [PubMed]

27. Senyuk, B.; Liu, Q.; Bililign, E.; Nystrom, P.D.; Smalyukh, I.I. Geometry-guided colloidal interactions and self-tiling of elastic dipoles formed by truncated pyramid particles in liquid crystals. Phys. Rev. E 2015, 91, 040501. [CrossRef] [PubMed]

28. Mikhael, J.; Roth, J.; Helden, L.; Bechinger, C. Archimedean-like tiling on decagonal quasicrystalline surfaces. Nature 2008, 454, 501. [CrossRef] [PubMed]

29. Luo, D.; Du, Q.G.; Dai, H.T.; Demir, H.V.; Yang, H.Z.; Ji, W.; Sun, X.W. Strongly linearly polarized low threshold lasing of all organic photonic quasicrystals. Sci. Rep. 2012, 2, 627. [CrossRef] [PubMed]

30. Tkalec, U.; Ravnik, M.; Čopar, S.; Z̆umer, S.; Muševič, I. Reconfigurable Knots and Links in Chiral Nematic Colloids. Science 2011, 333, 6038. [CrossRef] [PubMed]

31. Roichman, Y.; Grier, D.G. Holographic assembly of quasicrystalline photonic heterostructures. Opt. Exp. 2005, 13, 5434-5439. [CrossRef]

32. Ackerman, P.J.; Qi, Z.; Smalyukh, I.I. Optical generation of cyrstalline, quasicrystalline and arbitrary arrays of torons in confined cholesteric liquid crystals for patterning of optical vortices in laser beams. Phys. Rev. E 2012, 86, 021703. [CrossRef] [PubMed]

33. Stark, H. Physics of colloidal dispersions in nematic liquid crystals. Phys. Rep. 2001, 351, 387-474. [CrossRef]

34. Muševič, I.; S̆karabot, M.; Tkalec, U.; Ravnik, M.; Žumer, S. Two-Dimensional Nematic Colloidal Crystals Self-Assembled by Topological Defects. Science 2006, 313 954-958. [CrossRef] [PubMed]

35. Ravnik, M.; S̆karabot, M.; Žumer, S.; Tkalec, U.; Poberaj, I.; Babič, D.; Osterman, N.; Muševič, I. Entangled Nematic Colloidal Dimers and Wires. Phys. Rev. Lett. 2007, 99, 247801. [CrossRef] [PubMed]

36. Muševič, I. Liquid Crystal Colloids; Springer: Berlin, Germany, 2017.

37. Conradi, M.; Ravnik, M.; Bele, M.; Zorko, M.; Žumer, S.; Muševič, I. Janus nematic colloids. Soft Matter 2009, 5, 3905-3912. [CrossRef]

38. Liu, Q.; Senyuk, B.; Tasinkevych, M.; Smalyukh, I.I. Nematilc liquid crystal boojums with handles on colloidal handlbodies. Proc. Natl. Acad. Sci. USA 2013, 110, 9231-9236. [CrossRef] [PubMed]

39. Hashemi, S.M.; Jagodič, U.; Mozaffari, M.R.; Ejtehadi, M.R.; Muševič, I.; Ravnik, M. Fractal nematic colloids. Nat. Commun. 2017, 8, 14026. [CrossRef] [PubMed]

40. Silvestre, M.; Tasinkevych, M. Key-lock colloids in a nematic liquid crystal. Phys. Rev. E 2017, 95, 012606. [CrossRef] [PubMed]

41. Smalyukh, I.I.; Lansac, Y.; Clark, N.; Trivedi, R.P. Three-dimensional structure and multistable optical switching of triple-twisted particle-like excitations in anisotropic fluids. Nat. Mater. 2010, 9, 139-145. [CrossRef] [PubMed]

42. Varanytsia, A.; Posnjak, G.; Mur, U.; Joshi, V.; Darrah, K.; Muševič, I.; Čopar, S.; Chien, L. Topology-commanded optical properties of bistable electric-field-induced torons in cholesteric bubble domains. Sci. Rep. 2017, 7, 16149. [CrossRef] [PubMed]

43. Zumer, S.; Porenta, T.; Ravnik, M. Complex field-induced nematic defect structures in Laguerre-Gaussian optical tweezers. In Liquid Crystals XVI; SPIE: Bellingham, WA, USA, 2012; Volume 8475.

44. C̆opar, S. Topology and geometry of nematic braids. Phys. Rep. 2014, 538, 1-37. [CrossRef] 
45. Porenta, T.; Ravnik, M.; Žumer, S. Complex field-stabilised nematic defect structures in Laguerre-Gaussian optical tweezers. Soft Matter 2012, 8, 1865-1870. [CrossRef]

46. Damasceno, P.F.; Engel, M.; Glotzer, S.C. Predictive Self-Assembly of Polyhedra into Complex Structures. Science 2012, 337, 453-457. [CrossRef] [PubMed]

47. Cao, W.; Muoz, A.; Palffy-Muhoray, P.; Taheri, B. Lasing in a three-dimensional photonic crystal of the liquid crystal blue phase II. Nat. Mater. 2002, 1, 111. [CrossRef] [PubMed]

48. Mowatt, C.; Morrisa, S.M.; Wilkinson, T.D.; Coles, H.J. High slope efficiency liquid crystal lasers. Appl. Phys. Lett. 2010, 97, 251109. [CrossRef]

49. Gardiner, D.J.; Morris, S.M.; Hands, P.J.; Mowatt, C.; Rutledge, R.; Wilkinson, T.D.; Coles, H.J. Paintable band-edge liquid crystal lasers. Opt. Exp. 2011, 19, 2432-2439. [CrossRef] [PubMed]

50. Ko, D.H.; Morris, S.M.; Lorenz, A.; Castles, F.; Butt, H.; Gardiner, D.J.; Qasim, M.M.; Wallikewitz, B.; Hands, P.J.; Wilkinson, T.D.; et al. A nano-patterned photonic crystal laser with a dye-doped liquid crystal. Appl. Phys. Lett. 2013, 103, 051101. [CrossRef]

51. Stimulak, M.; Ravnik, M. Tunable photonic crystals with partial bandgaps from blue phase colloidal crystals and dielectric-doped blue phases. Soft Matter 2014,10 6339-6346. [CrossRef] [PubMed]

52. Alpinc, J.; Stimulak, M.; Čopar, S.; Ravnik, M. Nematic liquid crystal gyroids as photonic crystals. Liq. Cryst. 2016, 43, 2320-2331.

53. Humar, M.; Ravnik, M.; Pajk, S.; Muševič, I. Electrically tunable liquid crystal optical microresonators. Nat. Photonics 2009, 3, 595. [CrossRef]

54. Humar, M.; Muševic, I. 3D microlasers from self-assembled cholesteric liquid-crystal microdroplets. Opt. Exp. 2010, 18, 26995-27003. [CrossRef] [PubMed]

55. Peddireddy, K.; Jampani, V.S.R.; Thutupalli, S.; Herminghaus, S.; Bahr, C.; Muševič, I. Lasing and waveguiding in smectic A liquid crystal optical fibers. Opt. Exp. 2013, 21, 30233-30242. [CrossRef] [PubMed]

56. Ravnik, M.; S̆timulak, M.; Mur, U.; C̆anula, M.; Čopar, S.; Z̆umer, S. Photonic crystals, light manipulation, and imaging in complex nematic structures. In Emerging Liquid Crystal Technologies XI; International Society for Optics and Photonics: Bellingham, WA, USA, 2014.

57. Muševic, I. Integrated and topological liquid crystal photonics. Liq. Cryst. 2014, 41, 418-429. [CrossRef]

58. Alberucci, A.; Piccardi, A.; Bortolozzo, U.; Residori, S.; Assanto, G. Nematicon all-optical control in liquid crystal light valves. Opt. Lett. 2010, 35, 390-392. [CrossRef] [PubMed]

59. Hakobyan, D.; Brasselet, E. Left-handed optical radiation torque. Nat. Photonics 2014, 8, 610. [CrossRef]

60. Pile, D.; Brasselet, E. Backwards torque. Nat. Photonics 2014, 8, 664. [CrossRef]

61. Loussert, C.; Delabre, U.; Brasselet, E. Manipulating the Orbital Angular Momentum of Light at the Micron Scale with Nematic Disclinations in a Liquid Crystal Film. Phys. Rev. Lett. 2013, 111, 037802. [CrossRef] [PubMed]

62. C̆ančula, M.; Ravnik, M.; Žumer, S. Generation of vector beams with liquid crystal disclination lines. Phys. Rev. E 2014, 90, 022503. [CrossRef] [PubMed]

63. Kobashi, J.; Yoshida, H.; Ozaki, M. Planar optics with patterned chiral liquid crystals. Nat. Photonics 2016, 10, 389-392. [CrossRef]

64. Kosa, T.; Sukhomlinova, L.; Su, L.; Taheri, B.; White, T.J.; Bunning, T.J. Light-induced liquid crystallinity. Nature 2012, 485, 347-349. [CrossRef] [PubMed]

65. Yuan, Y.; Smalyukh, I.I. Topological nanocolloids with facile electric switching of plasmonic properties. Opt. Lett. 2015, 40, 5630-5633. [CrossRef] [PubMed]

66. Dontabhaktuni, J.; Madduri, S.; Bregar, A.; Ravnik, M. Self-organized three dimensional quasicrystals in nematic liquid crystals. 2018, submitted.

(c) 2018 by the authors. Licensee MDPI, Basel, Switzerland. This article is an open access article distributed under the terms and conditions of the Creative Commons Attribution (CC BY) license (http:/ / creativecommons.org/licenses/by/4.0/). 